\title{
On the classification of crepant analytically extremal contractions of smooth three-folds
}

\author{
Csilla Tamás
}

\begin{abstract}
We discuss the problem of classifying crepant analytically extremal contractions $X \rightarrow Y$ from a smooth 3-fold, contracting an irreducible normal divisor $D$ in $X$ to a point $P$ in $Y$. We prove that, if $D$ has degree $\left(-K_{D}\right)^{2} \geqslant 5$, the analytic structure of the contraction is completely determined by the isomorphism class of the exceptional locus and its normal bundle. This was previously known only for a smooth exceptional locus $D$.
\end{abstract}

\section{Introduction}

In the minimal model program, the study of certain types of birational contractions, called extremal, is of central importance. In [Mor82], S. Mori studied and classified birational extremal contractions $\varphi: X \rightarrow Y$ of smooth 3 -folds $X$ where the canonical bundle of $X$ is negative along the fibers of the contraction. His classification includes the following result: the exceptional locus $D=\operatorname{Exc}(\varphi)$ is an irreducible divisor. When $\operatorname{Exc}(\varphi)$ contracts to a curve on $Y, \operatorname{Exc}(\varphi)$ is a $\mathbb{P}^{1}$-bundle over the base curve and $Y$ is smooth. When $\operatorname{Exc}(\varphi)$ contracts to a point $q \in Y$, it is either $\mathbb{P}^{2}, \mathbb{P}^{1} \times \mathbb{P}^{1}$ or a singular quadric, with specified normal bundle, and $X$ is the blowup of $Y$ at $q$; in this case the analytic structure of the neighborhood of $\operatorname{Exc}(\varphi)$ is uniquely determined by the isomorphism class of $\operatorname{Exc}(\varphi)$ and its normal bundle in $X$. We call this feature the analytic rigidity of the contraction (see Definition 2.2).

In this paper we attempt to give a similar description for birational extremal contractions of smooth 3 -folds in the $K$-trivial case, i.e. when the relative canonical bundle $K_{X / Y}$ is numerically trivial (and hence the contraction is crepant); see $\S 2$ for a precise definition. Our main result is the following theorem.

Theorem 1.1 (Main Theorem). Let $X$ be a smooth projective 3 -fold over $\mathbb{C}$ and let $\varphi: X \rightarrow Y$ be a $K$-trivial birational extremal contraction onto a normal projective variety $Y$, contracting a divisor $D \subset X$ to a point $q \in Y$. Suppose $D$ is normal, and $\left(-K_{D}\right)^{2} \geqslant 5$. Then the contraction $\varphi$ is analytically rigid.

In this way we obtain the classification of $K$-trivial extremal contractions in terms of the exceptional divisor $D$ and its normal bundle $\mathcal{N}_{D / X}$ in $X$ in the case when $D$ is normal with $d=\left(-K_{D}\right)^{2} \geqslant 5$, contracting to a point. As the exceptional locus is a normal rational (possibly singular) del Pezzo surface $D$ of degree $d \geqslant 5$ with normal bundle isomorphic to $\mathcal{O}_{D}\left(K_{D}\right)$ (see $\S 2$ ), we obtain a finite list of possible contractions up to analytic isomorphism.

Received 5 July 2003, accepted in final form 23 July 2003, published online 15 October 2004. 2000 Mathematics Subject Classification 14E05, 14J30 (primary), 14E30, 14B10 (secondary). Keywords: 3-folds, birational, crepant, extremal, contractions, classification, del Pezzo surfaces.

This paper is based on the author's PhD thesis at Purdue University, written under the guidance of K. Matsuki. This journal is (C) Foundation Compositio Mathematica 2004. 


\section{Cs. TAmás}

As the analytic rigidity result shows, in order to know the analytic structure of the contraction, it is sufficient to construct one example for each possible exceptional locus. No algebraic examples are known except when $D$ is a non-singular del Pezzo surface of degree 6 [Nam97]. In $\S 6$ we present an example of an embedding of a singular del Pezzo surface of degree 7 into a smooth projective 3 -fold such that the canonical bundle of the ambient space is numerically trivial on $D$. By Fujiki's contraction theorem, $D$ can be contracted analytically, giving us a $K$-trivial contraction in the analytic category. Similar constructions can be carried out for each possible exceptional divisor $D$ (any normal rational del Pezzo surface of degree $d \geqslant 5$ ).

$K$-trivial extremal contractions have been studied by Gross [Gr97a] and Wilson [Wil97]. Their analysis of the case when the exceptional locus $D$ is a divisor contracting to a point includes a description of the (possible) exceptional loci, and the description of the analytic structure (and in particular establishing the analytic rigidity) when $D$ is non-singular with $\left(-K_{D}\right)^{2} \geqslant 5$. The analytic rigidity for $D$ non-normal or $\left(-K_{D}\right)^{2} \leqslant 4$ is not known. In fact, while the cubic hypersurface singularities $x^{3}+y^{3}+z^{3}+t^{3}+x y z t=0$ and $x^{3}+y^{3}+z^{3}+t^{3}=0$ are not isomorphic (see [MY82]), the exceptional locus of the blowup of the origin is the smooth cubic surface $x_{0}^{3}+x_{1}^{3}+x_{2}^{3}+x_{3}^{3}=0$ in both cases. On the other hand, if $D$ is non-normal, we have $\left(-K_{D}\right)^{2}=7$, and [Gr97b] gives a (local) example of a $K$-trivial contraction with exceptional divisor a non-normal del Pezzo surface of degree 7.

Wilson also describes the possible contractions if the exceptional locus $D$ contracts to a curve. Small $K$-trivial birational extremal contractions of smooth 3 -folds (i.e. when the exceptional locus is a collection of curves) are three-dimensional flopping contractions; these were studied in [Kol91] and [Rei83].

Even in the surface case, the condition of $K$-negativity or $K$-triviality is essential for analytic rigidity. In [Lau73], Laufer gives a complete list of taut surface singularities (i.e. normal two-dimensional singularities that are completely determined by the weighted dual graph $\Gamma$ of the exceptional locus $E$ of the minimal resolution). He also lists those singularities which are determined by the weighted dual graph and the analytic structure of $E$, and states that the singularities obtained by contractions of curves of general type are not determined by $\Gamma$ and the analytic structure of $E$. For example the singularity $x^{d}+y^{d}+z^{d}=0$ is not isomorphic to $x^{d}+y^{d}+z^{d}+f_{d+1}=0$ (where $f_{d+1}$ is a 'general' monomial of degree $d+1, d \geqslant 3$ ), whereas they both have the same exceptional curve and normal bundle.

We outline below the proof of the Main Theorem.

By [HR64, Theorem 3], the proof of analytic rigidity is reduced to showing that any two embeddings of $D$ into smooth complex 3-folds with normal bundles isomorphic to $\mathcal{O}_{D}\left(K_{D}\right)$ are formally equivalent. Suppose now that we have two $K$-trivial extremal contractions $\varphi: X \rightarrow Y$ and $\varphi^{\prime}: X^{\prime} \rightarrow Y^{\prime}$, with isomorphic exceptional divisors $D$ and $D^{\prime}$, and such that $\mathcal{N}_{D / X} \simeq \mathcal{N}_{D^{\prime} / X^{\prime}}$. To prove the formal equivalence, we first show that if $H^{1}\left(D, \mathcal{I}_{D} \otimes \mathcal{I}_{D} / \mathcal{I}_{D}^{2}\right)=0$, then the two embeddings $D \subset X$ and $D^{\prime} \subset X^{\prime}$ are 2-equivalent, i.e. we have an isomorphism of the ringed spaces $\left(D, \mathcal{O}_{X} / \mathcal{I}_{D}^{2}\right)$ and $\left(D^{\prime}, \mathcal{O}_{X^{\prime}} / \mathcal{I}_{D^{\prime}}^{2}\right)$, where $\mathcal{I}_{D}$ denotes the ideal sheaf of $D$ in $X$. Then we can obtain a formal equivalence by showing that the obstruction spaces $H^{1}\left(D, \mathcal{T}_{X} \otimes \mathcal{I}_{D}^{\nu} / \mathcal{I}_{D}^{\nu+1}\right)$ to extending a $\nu$-equivalence $(\nu \geqslant 2)$ to a $(\nu+1)$-equivalence vanish for all $\nu \geqslant 2$. The vanishing of both $H^{1}\left(D, \mathcal{T}_{D} \otimes \mathcal{I}_{D} / \mathcal{I}_{D}^{2}\right)$ and $H^{1}\left(D, \mathcal{T}_{X} \otimes \mathcal{I}_{D}^{\nu} / \mathcal{I}_{D}^{\nu+1}\right)$ is reduced to showing $H^{1}\left(D, \mathcal{T}_{D}\right)=0$ using properties of del Pezzo surfaces. This last vanishing is then proved using an explicit description of normal rational del Pezzo surfaces.

Remark 1.2. We should note that our results about formal equivalence hold over any algebraically closed field of characteristic 0 . However, over an arbitrary field there is no notion of analytic rigidity (and in particular we do not have Theorem 3 of [HR64]). Over an arbitrary field, formal equivalence implies only equivalence in the étale topology [Art69a, Theorem (4.6)]. 


\section{Classification of CREPANT EXTREMAL CONTRACTIONS}

\section{Conventions}

i) We are working over the field of complex numbers $\mathbb{C}$.

ii) We denote the tangent sheaf $\mathcal{H o m}_{\mathcal{O}_{D}}\left(\Omega_{D}^{1}, \mathcal{O}_{D}\right)$ of an algebraic variety $D$ by $\mathcal{T}_{D}$. The rest of our notations are standard in algebraic geometry.

\section{Preliminaries}

\section{1 $K$-trivial extremal contractions}

Definition 2.1. Let $X$ be a smooth projective $n$-fold over $\mathbb{C}$ and $\varphi: X \rightarrow Y$ a birational morphism onto a normal projective variety $Y$ such that the exceptional locus $D$ of $\varphi$ is of codimension 1 and such that $\operatorname{dim} \varphi(D)=0$. We call the contraction $\varphi$

P1) extremal if all the curves contracted by $\varphi$ are numerically proportional, i.e. given two curves $C$ and $C^{\prime}$ contracted by $\varphi$, there is a rational number $r$ such that for any divisor $E$ in $X$, we have $\left(E \cdot C^{\prime}\right)=r(E \cdot C)$,

P2) $K$-trivial if the canonical bundle on $X$ is numerically trivial on all curves contracted by $\varphi$, i.e. $\left(K_{X} \cdot C\right)=0$ for any curve $C$ contracted by $\varphi$ to a point.

Note that the condition P1 implies that the exceptional locus $D$ is an irreducible divisor (Proposition 2.4), and hence $\varphi$ contracts $D$ to a point $q \in Y$.

Definition 2.2. The contraction $\varphi$ is called analytically rigid if its analytic structure is uniquely determined by the isomorphism class of $\operatorname{Exc}(\varphi)=D$ and its normal bundle $\mathcal{N}_{D / X}$ in $X$. More precisely, suppose $\varphi^{\prime}: X^{\prime} \rightarrow Y^{\prime}$ is another birational map on a smooth projective 3 -fold $X^{\prime}$ with exceptional locus $D^{\prime}$, contracting $D^{\prime}$ to a point $q^{\prime} \in Y^{\prime}$. If $D \simeq D^{\prime}$ and we have an isomorphism of normal bundles $\mathcal{N}_{D / X} \simeq \mathcal{N}_{D^{\prime} / X^{\prime}}$, then the analytic rigidity of $\varphi$ means that there are open (analytic) neighborhoods $U$ of $D$ in $X$ and $U^{\prime}$ of $D^{\prime}$ in $X^{\prime}$ over which the contractions $\varphi$ and $\varphi^{\prime}$ are analytically isomorphic, i.e. we have the following commutative diagram (in the analytic category).

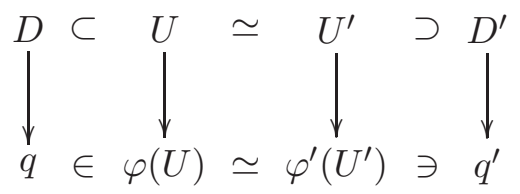

Remark 2.3. A priori our definition P1 of an extremal contraction is different from the one generally found in the literature, namely the 'contraction of an extremal ray'. However, by Proposition 2.4 below, P1 and P2 imply that the birational map $\varphi$ is the contraction of an extremal ray $R$ with respect to $K_{X}+\epsilon D$, for any $0<\epsilon$, where $R:=\mathbb{R}_{+}[C]$ for any curve $C \subset D$.

Proposition 2.4. Let $X$ be a non-singular projective variety of dimension $n$, and let $\varphi: X \rightarrow Y$ be a birational extremal contraction. Let $D$ denote the exceptional locus of $\varphi$ (with the reduced structure). Suppose that $\operatorname{codim}_{X} D=1$ and $\operatorname{dim} \varphi(D)=0$. Then $D$ is irreducible and $-D$ is $\varphi$-ample. Furthermore, $\varphi$ is the contraction of an extremal ray of the closure $\overline{N E}(X)$ of the cone of effective 1-cycles on $X$.

Proof. Suppose there are two distinct irreducible components $D_{1}$ and $D_{2}$ of $D$, and $\operatorname{codim}_{X} D_{1}=1$. Let $H_{1}, H_{2}, \ldots, H_{n-2} \subset X$ be general hyperplane sections of $X$. Let $H=\bigcap_{i=1}^{n-2} H_{i}$. Then $H$ is a smooth surface and $H \cap D_{1}$ is an irreducible curve $C_{1}$ on $D_{1}$. Then

$$
\left(D_{1} \cdot C_{1}\right)_{X}=\left(\left.D_{1}\right|_{H} \cdot C_{1}\right)_{H}=\left(C_{1}^{2}\right)_{H}<0
$$




\section{Cs. TAMÁs}

by the negativity of self-intersection of contractible curves. Now, if $C_{2} \subset D_{2}$ is a curve that is not contained in $D_{1}$, we have that $\left(D_{1} \cdot C_{2}\right) \geqslant 0$. But this contradicts the fact that $\varphi$ is extremal, because $C_{1}$ and $C_{2}$ cannot be numerically proportional. Therefore, $D$ is irreducible and for any curve $C \subset D$ we have $(D \cdot C)<0$.

In order to show that $-D$ is $\varphi$-ample, we only need to show that the divisor $-\left.D\right|_{D}$ is ample on $D$ [Gro61, Theorem III.4.7.1]. Then, by Kleiman's criterion of ampleness, it is enough to show that $(-D \cdot Z)>0$ for any $Z \in \overline{N E}(D)$.

The curves of $D$ are numerically proportional on $X$, therefore $Z \equiv r C_{1}$ on $X$ for some positive rational number $r$, since $Z \neq 0$ in $\overline{N E}(D)$. Therefore $(-D \cdot Z)=-r\left(D \cdot C_{1}\right)>0$, and hence $-D$ is relatively ample and the divisor $-\left.D\right|_{D}$ is ample on $D$.

In order to show that $\varphi$ is a contraction of an extremal ray, let $A$ be an ample divisor on $Y$. Then, by the contraction theorem of extremal rays [Mat01], the face $\left(\varphi^{*} A\right)^{\perp}$ of 1 -cycles intersecting trivially with $\varphi^{*} A$ in $\overline{N E}(X)$ contains an extremal ray $R$ (i.e. an edge of the cone $\overline{N E}(X)$ ) and we have a contraction $\operatorname{cont}_{R}: X \rightarrow Y^{\prime}$ of the extremal ray $R$.

But any curve $C$ in $R$ is contracted by $\varphi$, because $\left(\varphi^{*} A \cdot C\right)=0$. Therefore $C$ is numerically proportional to the curve $C_{1}$ in $D$, and hence the extremal ray $R$ is generated by $C_{1}$. Therefore $\varphi$ and $\operatorname{cont}_{R}$ contract the same curves. This implies $\varphi=\operatorname{cont}_{R}$.

Now we consider again our situation: let $X$ be a smooth projective 3 -fold and $\varphi: X \rightarrow Y$ a $K$-trivial birational extremal contraction, contracting a divisor $D$ to a point $q \in Y$. In fact, as our result concerns only the analytic structure of $\varphi$, we may drop the projectivity assumption on $X$, and study crepant contractions of smooth 3 -folds which are analytically extremal (i.e. have relative Picard number 1).

\subsection{Description of the exceptional divisor $D$}

By the adjunction formula,

$$
\left.\left.\mathcal{O}_{D}\left(-K_{D}\right) \simeq \mathcal{O}_{X}\left(-\left(K_{X}+D\right)\right)\right|_{D} \equiv \mathcal{O}_{X}(-D)\right|_{D},
$$

and hence $\mathcal{O}_{D}\left(-K_{D}\right)$ is ample on $D$, because $-D$ is $\varphi$-ample. Also, $D$ has only Gorenstein singularities, being a (Cartier) divisor on a smooth 3 -fold. Therefore $D$ is a so-called del Pezzo surface (i.e. Gorenstein with ample anticanonical bundle) of degree $d=\left(-K_{D}\right)^{2}$. Note that we allow the del Pezzo surface $D$ to be singular.

By [Gr97a, Theorem 5.2], the possibilities for the exceptional divisor $D$ are further restricted by its degree and singularities; $D$ is either

i) a normal and rational del Pezzo surface of degree $5 \leqslant d \leqslant 9$, or

ii) a non-normal del Pezzo surface of degree $d=7$, or

iii) a normal del Pezzo surface of degree $d \leqslant 4$ (rational for $d=4$ ).

In order to obtain information about the normal bundle $\mathcal{N}_{D / X}$ of $D$ in $X$, note that the equivalences (2.1) above also show that $\mathcal{N}_{D / X}$ is numerically equivalent to $\mathcal{O}_{D}\left(K_{D}\right)$. In fact, we have that $\mathcal{N}_{D / X} \simeq \mathcal{O}_{D}\left(K_{D}\right)$ (using that the Euler characteristic is a numerical invariant [Gro67b, Corollary 09] and that $\chi\left(\mathcal{O}_{D}\right)=1$ for del Pezzo surfaces [HW81]).

\subsection{The singularity at $q \in Y$}

Because $\varphi: X \rightarrow Y$ is $K$-trivial and extremal, the singularity $q \in Y$ is a rational Gorenstein singularity (i.e. it is Gorenstein, and $\varphi_{*} K_{X}=K_{Y}$ ). According to [Rei79], to an isolated rational Gorenstein 3-fold point $q \in Y$ one can attach a natural number $k \geqslant 0$ such that: 


\section{Classification of CREPANT EXTREMAl CONTRACTIONS}

i) if $k=0$ then $q \in Y$ is a $\mathrm{cDV}$ point;

ii) if $k=1$ then $q \in Y$ is a hypersurface singularity that is locally of the form $x^{2}+y^{3}+f(y, z, t)=0$, where $f=y f_{1}(z, t)+f_{2}(z, t)$ and $f_{1}$ (respectively $f_{2}$ ) is a sum of monomials $z^{a} t^{b}$ of degree $a+b \geqslant 4$ (respectively $\geqslant 6$ );

iii) if $k=2$ then $q \in Y$ is a hypersurface singularity that is locally of the form $x^{2}+f(y, z, t)=0$, where $f$ is a sum of monomials of degree $\geqslant 4$;

iv) if $k \geqslant 3$ then mult $_{q} Y=k$ and the embedding dimension of $q \in Y$ is $k+1$; in particular, for $k=3, q \in Y$ is still a hypersurface singularity, and for $k=4$ it is a complete intersection.

Remark 2.5 [Rei79, Proposition (2.13)]. Reid implies that, if the exceptional locus of the map $\varphi: X \rightarrow Y$ is a del Pezzo surface of degree $d$, then the invariant $k$ is equal to $d$. We also have that $X$ is the (weighted) blowup of $Y$ at $q$ [Rei79, Theorem (2.11)]. In particular, when $d \geqslant 3, X$ is the blowup of $Y$ at $q$.

\subsection{Normal rational del Pezzo surfaces of degree $\geqslant 5$}

Definition 2.6. A two-dimensional (possibly singular) projective variety $D$ is called a del Pezzo surface if it has only Gorenstein singularities, and its anticanonical sheaf $\mathcal{O}_{D}\left(-K_{D}\right)$ is ample. We call the intersection number $d=\left(-K_{D}\right)^{2}$ the degree of the del Pezzo surface $D$.

Normal rational del Pezzo surfaces were classified by Hidaka and Watanabe in [HW81]; non-normal ones by Reid in [Rei94]. In this section we enumerate some facts that will be used subsequently.

Let $D$ be a normal del Pezzo surface of degree $d$ and $\pi: \tilde{D} \rightarrow D$ a minimal resolution of $D$. Then $D$ is either a cone over an elliptic curve, or it is rational. In the latter case, $D$ is either $\mathbb{P}^{2}$ $(d=9), \mathbb{P}^{1} \times \mathbb{P}^{1}(d=8)$, a singular quadric in $\mathbb{P}^{3}(d=8)$, or its minimal resolution $\tilde{D}$ is the blowup of $9-d$ points $\Sigma$ in almost general position on $\mathbb{P}^{2}$ [HW81].

Up to projective automorphisms of $\mathbb{P}^{2}$ (and their extensions to the blowup spaces), there are 22 different configurations of at most four points in almost general position (including $\Sigma=\emptyset$, when $D \simeq \mathbb{P}^{2}$ ), so there are 22 non-isomorphic rational del Pezzo surfaces of degree $d \geqslant 5$ other than $\mathbb{P}^{1} \times \mathbb{P}^{1}$ or a singular quadric. The following is a well known lemma.

Lemma 2.7. A normal rational del Pezzo surface $D$ of degree $d \geqslant 5$ is either non-singular, or it can have only the following singularities: $A_{1}, 2 A_{1}, A_{2}, A_{1} A_{2}, A_{3}$ or $A_{4}$.

Note that for $d \geqslant 3$, the anticanonical sheaf $\mathcal{O}_{D}\left(-K_{D}\right)$ is very ample and its global sections yield an embedding of $D$ into $\mathbb{P}^{d}$ as a subvariety of degree $d$. This embedding defines a projectively normal variety and is defined by quadric equations except for the case $d=3$ [HW81, Theorem 4.4].

Proposition 2.8 [HW81, Proposition 4.2]. Let D be a normal del Pezzo surface. Then the following hold:

i) the anticanonical system $\left|-K_{D}\right|$ of $D$ contains a non-singular elliptic curve;

ii) $H^{1}\left(D, \mathcal{O}_{D}\left(\nu K_{D}\right)\right)=0$ for all $\nu \in \mathbb{Z}$;

iii) if $\operatorname{deg} D=d$, then

$$
\operatorname{dim} H^{0}\left(D, \mathcal{O}_{D}\left(-\nu K_{D}\right)\right)= \begin{cases}\frac{(\nu+1) \nu}{2} d+1 & \text { if } \nu \geqslant 0 \\ 0 & \text { if } \nu<0 .\end{cases}
$$




\section{Cs. TAMÁs}

\section{Analytic equivalence, formal equivalence and infinitesimal extensions}

\subsection{Analytic versus formal equivalence}

A standard tool for showing analytic equivalence is a criterion due to Grauert [Gra62] and Hironaka and Rossi [HR64] that reduces the problem of showing analytic equivalence of embeddings to that of showing formal equivalence [HR64, Theorem 3]. A careful reading of the proof of [HR64, Lemma 9] gives us the following lemma.

Lemma 3.1. Let $X$ be a non-singular complex manifold of dimension $n$ and let $D$ be a complex subspace with ideal sheaf $\mathcal{I}_{D}$. We assume that either $D$ is reduced, or $D$ is a (Cartier) divisor. Suppose that there exists an integer $\nu_{0} \geqslant 2$ such that $H^{1}\left(D, \mathcal{T}_{X} \otimes \mathcal{I}_{D}^{\nu} / \mathcal{I}_{D}^{\nu+1}\right)=0$ for any $\nu \geqslant \nu_{0}$. Then a $\nu$-equivalence $\left(\nu \geqslant \nu_{0}\right)$ of $D$ with a complex subspace $D^{\prime}$ of a complex space $X^{\prime}$, where $X^{\prime}$ has the same dimension as $X$ at all points of $D^{\prime}$, extends to a formal equivalence.

By $\nu$-equivalence we mean an isomorphism of the complex spaces $\left(D, \mathcal{O}_{X} / \mathcal{I}_{D}^{\nu}\right)$ and $\left(D^{\prime}, \mathcal{O}_{X^{\prime}} / \mathcal{I}_{D^{\prime}}^{\nu}\right)$, where $\mathcal{I}_{D}$, respectively, $\mathcal{I}_{D^{\prime}}$ is the ideal sheaf of $D$ in $X$, respectively of $D^{\prime}$ in $X^{\prime}$. Formal equivalence means an isomorphism of the completions:

$$
\hat{X}=\lim _{\longleftarrow}\left(D, \mathcal{O}_{X} / \mathcal{I}_{D}^{\nu}\right) \simeq \hat{X}^{\prime}=\lim _{\longleftarrow}\left(D^{\prime}, \mathcal{O}_{X^{\prime}} / \mathcal{I}_{D^{\prime}}^{\nu}\right) .
$$

Remark 3.2. For any $\nu$, the obstruction to extending a $\nu$-equivalence to a $(\nu+1)$-equivalence lies in the cohomology group $H^{1}\left(D, \mathcal{T}_{X} \otimes \mathcal{I}_{D}^{\nu} / \mathcal{I}_{D}^{\nu+1}\right)$.

In our case we are given a divisor $D$ in a smooth 3 -fold $X$, with given normal bundle $\mathcal{N}_{D / X}$ (which is isomorphic to $\mathcal{O}_{D}\left(K_{D}\right)$ ). In general, if we have an embedding of any scheme $D$ into a scheme $X$ such that $D$ has conormal sheaf $\mathcal{L}$ in $X$, then we have the exact sequence

$$
0 \rightarrow \mathcal{L} \rightarrow \mathcal{O}_{2 D} \rightarrow \mathcal{O}_{D} \rightarrow 0,
$$

where $2 D$ is the 2 -structure on $D$ obtained from the embedding $D \subset X$. Therefore $2 D$ is an infinitesimal extension of $D$ by the sheaf $\mathcal{L}$ (i.e. $\mathcal{L}$ can be considered as an ideal sheaf with square 0 on the scheme $2 D$, with $\mathcal{O}_{2 D} / \mathcal{L} \simeq \mathcal{O}_{D}$ [Har77, Exercise II.8.7]; see also [Gro64, § 18]). Below (see Proposition 3.5) we show that, if $H^{1}\left(D, \mathcal{T}_{D} \otimes \mathcal{L}\right)=0$, then $D$ uniquely extends to $2 D$ with the given conormal sheaf $\mathcal{L}$. As we see in $\S 4$, this condition is satisfied in our case and hence we may apply Lemma 3.1 with $\nu_{0}=2$.

\subsection{Certain local conditions}

Lemma 3.3. Let $D$ be a (not necessarily reduced) divisor in a smooth $n$-fold $X$, and let $\mathcal{L} \simeq \mathcal{O}_{D}(-D)$ be the conormal bundle of $D$ in $X$. Then the scheme $2 D$ is locally uniquely determined by $D$ and $\mathcal{L}$. More precisely, if we have another embedding $D \simeq D^{\prime} \subset X^{\prime}$ into a smooth $n$-fold $X^{\prime}$ such that $\left(\mathcal{N}_{D^{\prime} / X^{\prime}}\right)^{*} \simeq \mathcal{L}$, then the schemes $2 D$ and $2 D^{\prime}$ are locally isomorphic.

Proof. As noted before, both $2 D$ and $2 D^{\prime}$ are infinitesimal extensions of $D$ by $\mathcal{L}$. The question is local, so we may assume that $X=\operatorname{Spec} R, R$ a regular ring, $D=\operatorname{Spec}(R / f)$ (and hence $2 D=$ $\left.\operatorname{Spec}\left(R / f^{2}\right)\right)$ and $2 D^{\prime}=\operatorname{Spec} A$, where the support of $A$ is $D$, and at any point, the embedding dimension of $A$ is the same as the embedding dimension of $D$. So we have two infinitesimal extensions of $R / f$ by $R / f$ :

$$
0 \rightarrow R / f \stackrel{\alpha}{\rightarrow} R / f^{2} \stackrel{\beta}{\rightarrow} R / f \rightarrow 0 \quad \text { and } \quad 0 \rightarrow R / f \stackrel{\tilde{\alpha}}{\rightarrow} A \stackrel{\tilde{\beta}}{\rightarrow} R / f \rightarrow 0 .
$$




\section{Classification of CREPANT eXtremal CONTRACTIONS}

The ring homomorphism $R / f^{2} \rightarrow R / f$ factors through $A$ [Har77, Exercise II.8.6], and thus we have the following commutative diagram.

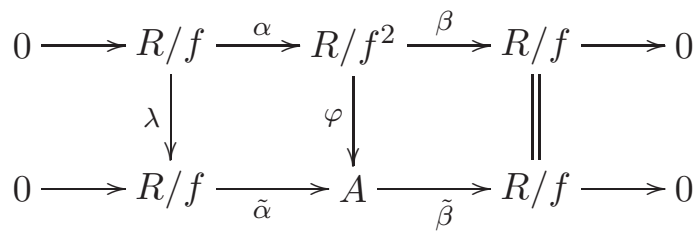

From here, we obtain an isomorphism of extensions

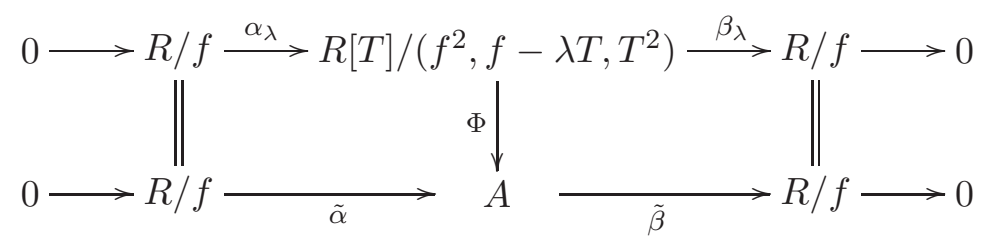

where $\alpha_{\lambda}(r)=r T, \beta_{\lambda}(r+s T)=\beta(r)$, and $\Phi$ is a ring homomorphism defined by $\Phi(r+s T)=$ $\varphi(r)+\tilde{\alpha}(s \bmod f)$; we regard $\lambda$ as an element of $R$.

As all infinitesimal extensions of non-singular affine schemes are trivial, it is sufficient to consider the case when $R$ is local of dimension $n, D=\operatorname{Spec}(R / f)$ is $\operatorname{singular}$, and so $\operatorname{embdim}(R / f)=n$.

By completion, we may assume $R \simeq \mathbb{C}\left[\left[X_{1}, X_{2}, \ldots, X_{n}\right]\right]$. However, the embedding dimension of $\mathbb{C}\left[\left[X_{1}, X_{2}, \ldots, X_{n}, T\right]\right] /\left(f^{2}, f-\lambda T, T^{2}\right)$ is $n$ if and only if $\lambda$ is a unit. But then (3.1) implies that the completion $\hat{\varphi}$ of $\varphi$ is an isomorphism, and (as completion is faithfully flat) that $\varphi: R / f^{2} \rightarrow A$ is an isomorphism. So, although the infinitesimal extension $0 \rightarrow R / f \rightarrow A \rightarrow R / f \rightarrow 0$ is not unique, the $\operatorname{ring} A$ is.

Remark 3.4. The same statement is true if we only assume that $D$ is a $(n-1)$-dimensional scheme which is locally a divisor in a smooth $n$-fold, and that there exists a scheme $2 D$ in which $D$ has conormal bundle $\mathcal{L}$.

\subsection{Cohomological condition for the (global) uniqueness of $\mathcal{O}_{2 D}$}

In what follows, we show that, if we fix the local data given by an infinitesimal extension of $D$ by $\mathcal{L}$, then the set of all (isomorphism classes of) extensions with the same local data is in one-to-one correspondence with $H^{1}\left(D, \mathcal{T}_{D} \otimes \mathcal{L}\right)$.

More precisely, let now $\left(D, \mathcal{O}_{D}\right)$ be a ringed space, $\mathcal{L}$ a sheaf of $\mathcal{O}_{D}$-modules, and consider the following local data: for any $p \in D$, an infinitesimal extension $0 \rightarrow L_{p} \stackrel{\alpha_{p}}{\longrightarrow} A_{p} \stackrel{\beta_{p}}{\longrightarrow} B_{p} \rightarrow 0$, where $L_{p}=\mathcal{L}_{p}$ and $B_{p}=\mathcal{O}_{D, p}$. We say that an infinitesimal extension $0 \rightarrow \mathcal{L} \stackrel{\alpha}{\rightarrow} \mathcal{A} \stackrel{\beta}{\rightarrow} \mathcal{O}_{D} \rightarrow 0$ of $D$ by $\mathcal{L}$ has the given local data $\left(A_{p}, \alpha_{p}, \beta_{p}\right)$ if, for all $p \in D$, we have isomorphism of extensions as follows.

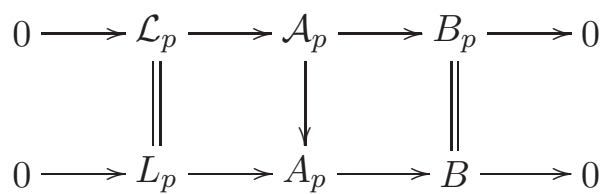

Proposition 3.5. Suppose we have a ringed space $\left(D, \mathcal{O}_{D}\right)$, a sheaf $\mathcal{L}$ of $\mathcal{O}_{D}$-modules, and an infinitesimal extension $(\mathcal{A}, \alpha, \beta)$ of $D$ by $\mathcal{L}$ with local data $\left(\mathcal{A}_{p}, \alpha_{p}, \beta_{p}\right)$. Then the set of isomorphism classes of infinitesimal extensions of $D$ by $\mathcal{L}$ having the local data $\left(\mathcal{A}_{p}, \alpha_{p}, \beta_{p}\right)$ is in one-to-one correspondence with

$$
H^{1}\left(D, \operatorname{Der}\left(\mathcal{O}_{D}, \mathcal{L}\right)\right)=H^{1}\left(D, \mathcal{H o m}_{D}\left(\Omega_{D}^{1}, \mathcal{L}\right)\right) .
$$

Remark 3.6. This result was stated (without details) in [Rei75]. 


\section{Cs. TAMÁs}

Proof of Proposition 3.5. Let $0 \rightarrow \mathcal{L} \stackrel{\alpha}{\rightarrow} \mathcal{A} \stackrel{\beta}{\rightarrow} \mathcal{O}_{D} \rightarrow 0$ be the given infinitesimal extension with local data $\left(\mathcal{A}_{p}, \alpha_{p}, \beta_{p}\right)$. Cover $D$ with affine open sets $V_{i}=\operatorname{Spec} B_{i}$, and denote $\left.\mathcal{L}\right|_{V_{i}}=L_{i},\left.\mathcal{A}\right|_{V_{i}}=A_{i}$.

Given another extension $0 \rightarrow \mathcal{L} \stackrel{\tilde{\alpha}}{\rightarrow} \tilde{\mathcal{A}} \stackrel{\tilde{\beta}}{\rightarrow} \mathcal{O}_{D} \rightarrow 0$ with local data $\left(\mathcal{A}_{p}, \alpha_{p}, \beta_{p}\right)$, on $V_{i}$ we have the following isomorphisms of extensions.

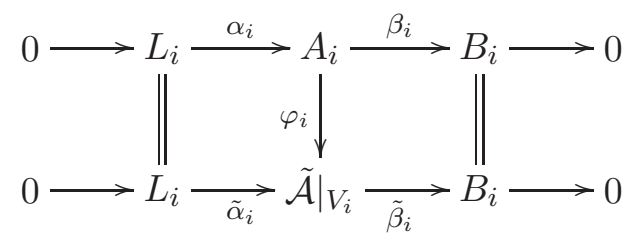

On the intersections $V_{i j}=V_{i} \cap V_{j}$, the morphisms $\varphi_{i}$ and $\varphi_{j}$ differ by a derivation: if we denote $\varphi_{i j}=\varphi_{j}^{-1} \circ \varphi_{i}$, then $\varphi_{i j}(a)=a+\theta_{i j}(\beta(a))$, where $\theta_{i j} \in \operatorname{Der}_{k}\left(B_{i j}, L_{i j}\right)$. Thus the extension $\tilde{\mathcal{A}}$ gives a (well-defined) cohomology class $\left[\left\{\theta_{i j}\right\}\right] \in \check{H}^{1}\left(\left\{V_{i}\right\}, \operatorname{Der}\left(\mathcal{O}_{D}, \mathcal{L}\right)\right) \simeq H^{1}\left(D, \mathcal{H o m}\left(\Omega_{D}^{1}, \mathcal{L}\right)\right)$.

Conversely, given a cohomology class $\left[\left\{\theta_{i j}\right\}\right] \in \check{H}^{1}\left(\left\{V_{i}\right\}, \operatorname{Der}\left(\mathcal{O}_{D}, \mathcal{L}\right)\right)$, we obtain isomorphisms of extensions $\varphi_{i j}:\left(\left.\mathcal{A}\right|_{V_{i j}}, \alpha_{i j}, \beta_{i j}\right) \rightarrow\left(\left.\mathcal{A}\right|_{V_{i j}}, \alpha_{i j}, \beta_{i j}\right)$. We define the infinitesimal extension $\tilde{\mathcal{A}}$ by

$$
\tilde{\mathcal{A}}(V)=\left\{\left(s_{i}\right)_{i \in I} \in \prod \mathcal{A}\left(V_{i} \cap V\right): \varphi_{i j}\left(\left.s_{i}\right|_{\left(V_{i j} \cap V\right)}\right)=\left.s_{j}\right|_{V_{i j}}\right\}
$$

Remark 3.7. Similar proof shows that extensions of $\nu D$ to $(\nu+1) D$ are in one-to-one correspondence with $H^{1}\left(D, \mathcal{H o m}_{\nu D}\left(\Omega_{\nu D}^{1}, \mathcal{L}\right)\right)$.

Corollary 3.8. Let $D$ be a (not necessarily reduced) divisor in a smooth $n$-fold $X$, and let $\mathcal{L}$ be the conormal bundle of $D$ in $X$. Suppose that $H^{1}\left(D, \mathcal{T}_{D} \otimes \mathcal{L}\right)=0$. Then $\mathcal{O}_{2 D}$ is unique up to isomorphism.

\section{Reducing the proof to the vanishing of $H^{1}\left(D, \mathcal{T}_{D}\right)$}

We now return to the proof of the Main Theorem, restated as follows.

Theorem 4.1 (Main Theorem). Let $X$ be a smooth 3-fold over $\mathbb{C}$ and let $\varphi: X \rightarrow Y$ be a crepant analytically extremal contraction, with exceptional locus $D$ a normal rational del Pezzo surface of degree $d \geqslant 5$, contracting to a point $q \in Y$. Then the contraction $\varphi$ is analytically rigid.

As we noted in $\S 2$, a normal rational del Pezzo surface of degree $d \geqslant 5$ has only $A_{n}$-type singularities (Lemma 2.7), and $\mathcal{N}_{D / X} \simeq \mathcal{O}_{D}\left(K_{D}\right)$. If we denote by $\mathcal{I}_{D}$ the ideal sheaf of $D$ in $X$, then

$$
\mathcal{I}_{D}^{\nu} / \mathcal{I}_{D}^{\nu+1} \simeq\left(\mathcal{I}_{D} / \mathcal{I}_{D}^{2}\right)^{\otimes \nu} \simeq \mathcal{O}_{D}\left(-\nu K_{D}\right)
$$

Based on the results of $\S 3$, the proof of the Main Theorem is reduced to showing the vanishing of the cohomology groups $H^{1}\left(D, \mathcal{T}_{D} \otimes \mathcal{I}_{D} / \mathcal{I}_{D}^{2}\right)$ and $H^{1}\left(D, \mathcal{T}_{X} \otimes \mathcal{I}_{D}^{\nu} / \mathcal{I}_{D}^{\nu+1}\right)$, for all $\nu \geqslant 2$. In this section we show that $H^{1}\left(D, \mathcal{T}_{D}\right)=0$ is a sufficient condition for achieving these vanishings, and we defer the proof of the following theorem till $\S 5$.

Theorem 4.2. Let $D$ be a normal rational del Pezzo surface of degree $d \geqslant 5$. Then

$$
H^{1}\left(D, \mathcal{T}_{D}\right)=0 \text {. }
$$

Proposition 4.3. Let $D$ be a normal rational del Pezzo surface of degree $d \geqslant 5$. Then we have

$$
H^{1}\left(D, \mathcal{T}_{D} \otimes \mathcal{O}_{D}\left(-\nu K_{D}\right)\right)=0 \text { for all } \nu \geqslant 0 .
$$




\section{Classification of CREPANT EXTREMAL CONTRACTIONS}

Proof. Let $H \in\left|-K_{D}\right|$ be a general hyperplane section; we can take it to be an irreducible, smooth elliptic curve (Proposition 2.8) that avoids the singular points of $D$.

Note that $\mathcal{T}_{D}$ is locally free outside the singular points of $D$, and therefore tensoring

$$
0 \rightarrow \mathcal{O}_{D}(-H) \rightarrow \mathcal{O}_{D} \rightarrow \mathcal{O}_{H} \rightarrow 0
$$

by $\mathcal{T}_{D} \otimes \mathcal{O}_{D}\left(-\nu K_{D}\right)$ we obtain

$$
\left.0 \rightarrow \mathcal{T}_{D} \otimes \mathcal{O}_{D}\left((1-\nu) K_{D}\right) \rightarrow \mathcal{T}_{D} \otimes \mathcal{O}_{D}\left(-\nu K_{D}\right) \rightarrow \mathcal{T}_{D} \otimes \mathcal{O}_{D}\left(-\nu K_{D}\right)\right|_{H} \rightarrow 0 .
$$

Next we consider the exact sequence

$$
0 \rightarrow \mathcal{I}_{H} /\left.\mathcal{I}_{H}^{2} \rightarrow \Omega_{D}^{1}\right|_{H} \rightarrow \Omega_{H}^{1} \rightarrow 0,
$$

where $\mathcal{I}_{H}$ is the ideal sheaf of $H$ in $D$. Since $H$ is an elliptic curve and $\operatorname{deg}\left(-\left.K_{D}\right|_{H}\right)=d=\operatorname{deg}(D)$, we obtain that

$$
\begin{aligned}
& h^{0}\left(H,\left.\mathcal{T}_{D} \otimes \mathcal{O}_{D}\left(-\nu K_{D}\right)\right|_{H}\right)=(2 \nu+1) d, \\
& h^{1}\left(H,\left.\mathcal{T}_{D} \otimes \mathcal{O}_{D}\left(-\nu K_{D}\right)\right|_{H}\right)=0 .
\end{aligned}
$$

Therefore, by induction, using the long exact sequence associated to (4.3) and the condition $H^{1}\left(D, \mathcal{T}_{D}\right)=0$, we obtain $H^{1}\left(D, \mathcal{T}_{D} \otimes \mathcal{O}_{D}\left(-\nu K_{D}\right)\right)=0$ and $H^{2}\left(D, \mathcal{T}_{D} \otimes \mathcal{O}_{D}\left(-\nu K_{D}\right)\right)=0$ for all $\nu \geqslant 0$. This completes the proof of Proposition 4.3.

ThEOREM 4.4. Under the assumptions of the Main Theorem, we have

$$
H^{1}\left(D, \mathcal{T}_{X} \otimes \mathcal{I}_{D}^{\nu} / \mathcal{I}_{D}^{\nu+1}\right)=0, \quad \text { for all } \nu \geqslant 2
$$

Proof. When $D$ is non-singular, the theorem is an easy consequence of Proposition 4.3 and the vanishing of the first cohomology of $\mathcal{O}_{D}\left((1-\nu) K_{D}\right)$ for any del Pezzo surface (Proposition 2.8). We actually obtain the vanishing of $H^{1}\left(D, \mathcal{T}_{X} \otimes \mathcal{I}_{D}^{\nu} / \mathcal{I}_{D}^{\nu+1}\right)=0$, for all $\nu \geqslant 1$.

So we concentrate on the case when $D$ is normal rational. In this case the difficulty comes from the fact that the tangent sheaf $\mathcal{T}_{D}$ is not locally free any more. In fact, $H^{1}\left(D, \mathcal{T}_{X} \otimes \mathcal{I}_{D} / \mathcal{I}_{D}^{2}\right)$ does not vanish in general (see Remark 4.6 below), but an easy argument shows that it is enough to prove that $H^{1}\left(D, \mathcal{T}_{X} \otimes \mathcal{I}_{D}^{2} / \mathcal{I}_{D}^{3}\right)=0$.

Indeed, for a general $H \in\left|-K_{D}\right|$, as in the proof of Proposition 4.3, tensor

$$
0 \rightarrow \mathcal{O}_{D}(-H) \rightarrow \mathcal{O}_{D} \rightarrow \mathcal{O}_{H} \rightarrow 0
$$

by $\mathcal{T}_{X} \otimes \mathcal{I}_{D}^{\nu} / \mathcal{I}_{D}^{\nu+1}$ for some $\nu \geqslant 2$. From the long exact sequence of cohomology, we get

$$
H^{1}\left(D, \mathcal{T}_{X} \otimes \mathcal{I}_{D}^{\nu-1} / \mathcal{I}_{D}^{\nu}\right) \rightarrow H^{1}\left(D, \mathcal{T}_{X} \otimes \mathcal{I}_{D}^{\nu} / \mathcal{I}_{D}^{\nu+1}\right) \rightarrow H^{1}\left(H, \mathcal{T}_{X} \otimes \mathcal{I}_{D}^{\nu} /\left.\mathcal{I}_{D}^{\nu+1}\right|_{H}\right)
$$

Consider the exact sequences

$$
\left.0 \rightarrow \mathcal{T}_{H} \rightarrow \mathcal{T}_{X}\right|_{H} \rightarrow \mathcal{N}_{H / X} \rightarrow 0
$$

and

$$
\left.0 \rightarrow \mathcal{N}_{H / D} \rightarrow \mathcal{N}_{H / X} \rightarrow \mathcal{N}_{D / X}\right|_{H} \rightarrow 0
$$

The latter sequence is exact because both $H$ in $D$ and $D$ in $X$ are Cartier divisors; see for example [Gro67a, 19.1.5].

We know that $\mathcal{N}_{H / D}=\mathcal{O}_{H}\left(-K_{D}\right),\left.\mathcal{N}_{D / X}\right|_{H}=\mathcal{O}_{H}\left(K_{D}\right)$, so tensoring the above exact sequences with $\mathcal{I}_{D}^{\nu} / \mathcal{I}_{D}^{\nu+1}=\mathcal{O}_{D}\left(-\nu K_{D}\right)$ yields $H^{1}\left(H, \mathcal{N}_{H / X} \otimes \mathcal{I}_{D}^{\nu} / \mathcal{I}_{D}^{\nu+1}\right)=0$ (because $-K_{D}$ is non-zero and effective), and hence $H^{1}\left(H, \mathcal{T}_{X} \otimes \mathcal{I}_{D}^{\nu} /\left.\mathcal{I}_{D}^{\nu+1}\right|_{H}\right)=0$ for all $\nu \geqslant 2$. (Recall that $H$ is an elliptic curve.) 


\section{Cs. TAmás}

Therefore we have surjections

$$
H^{1}\left(D, \mathcal{T}_{X} \otimes \mathcal{I}_{D}^{\nu-1} / \mathcal{I}_{D}^{\nu}\right) \rightarrow H^{1}\left(D, \mathcal{T}_{X} \otimes \mathcal{I}_{D}^{\nu} / \mathcal{I}_{D}^{\nu+1}\right) \rightarrow 0 \quad \forall \nu \geqslant 2,
$$

and so we can use induction on $\nu$ to prove the vanishing of the groups $H^{1}\left(D, \mathcal{T}_{X} \otimes \mathcal{I}_{D}^{\nu} / \mathcal{I}_{D}^{\nu+1}\right)$.

Next we show that $H^{1}\left(D, \mathcal{T}_{X} \otimes \mathcal{I}_{D}^{2} / \mathcal{I}_{D}^{3}\right)=0$. The sequence

$$
0 \rightarrow \mathcal{I}_{D} /\left.\mathcal{I}_{D}^{2} \rightarrow \Omega_{X}^{1}\right|_{D} \rightarrow \Omega_{D}^{1} \rightarrow 0
$$

is exact because $D$ is a Cartier divisor in the smooth 3 -fold $X$. The dual of this sequence gives us

$$
\left.0 \rightarrow \mathcal{T}_{D} \rightarrow \mathcal{T}_{X}\right|_{D} \rightarrow\left(\mathcal{I}_{D} / \mathcal{I}_{D}^{2}\right)^{*} \otimes \mathcal{I}_{S(D)} \rightarrow 0
$$

where $S(D)$ is the scheme of singularities of $D$. We tensor this with $\mathcal{I}_{D}^{2} / \mathcal{I}_{D}^{3} \simeq \mathcal{O}_{D}\left(-2 K_{D}\right)$; the corresponding long exact sequence on cohomology gives

$$
\begin{aligned}
0=H^{1}\left(D, \mathcal{T}_{D} \otimes \mathcal{I}_{D}^{2} / \mathcal{I}_{D}^{3}\right) & \rightarrow H^{1}\left(D, \mathcal{I}_{X} \otimes \mathcal{I}_{D}^{2} / \mathcal{I}_{D}^{3}\right) \\
& \rightarrow H^{1}\left(D, \mathcal{O}_{D}\left(-K_{D}\right) \otimes \mathcal{I}_{S(D)}\right) \rightarrow H^{2}\left(D, \mathcal{T}_{D} \otimes \mathcal{I}_{D}^{2} / \mathcal{I}_{D}^{3}\right)=0 .
\end{aligned}
$$

Therefore $H^{1}\left(D, \mathcal{T}_{X} \otimes \mathcal{I}_{D}^{2} / \mathcal{I}_{D}^{3}\right) \simeq H^{1}\left(D, \mathcal{I}_{S(D)}\left(-K_{D}\right)\right)$. We now show that $H^{1}\left(D, \mathcal{I}_{S(D)}\left(-K_{D}\right)\right)=0$.

From the exact sequence

$$
0 \rightarrow \mathcal{I}_{S(D)} \rightarrow \mathcal{O}_{D} \rightarrow \mathcal{O}_{S(D)} \rightarrow 0
$$

we obtain that $H^{1}\left(D, \mathcal{I}_{S(D)}\left(-K_{D}\right)\right)=\operatorname{coker}\left(\Phi_{D}: H^{0}\left(D, \mathcal{O}_{D}\left(-K_{D}\right)\right) \rightarrow H^{0}\left(S(D), \mathcal{O}_{S(D)}\left(-K_{D}\right)\right)\right)$.

Suppose that the singularities of $D$ are $p_{1}, p_{2}, \ldots, p_{r}$, of type $A_{\lambda_{1}}, A_{\lambda_{2}}, \ldots, A_{\lambda_{r}}$ respectively. Then $h^{0}\left(S(D), \mathcal{O}_{S(D)}\left(-K_{D}\right)\right)=\sum_{i=1}^{r} \lambda_{i}$. We also know that $h^{0}\left(D, \mathcal{O}_{D}\left(-K_{D}\right)\right)=d+1$, where $d=\operatorname{deg} D$.

Claim. We claim that $\operatorname{dim} \operatorname{ker}\left(\Phi_{D}\right)=d+1-\sum_{i=1}^{r} \lambda_{i}$.

Note that as $D$ has only isolated singularities $p_{1}, p_{2}, \ldots, p_{r}, \mathcal{O}_{S(D)}$ is the direct sum of the Milnor algebras $\mathcal{O}_{D, p_{i}} / J\left(p_{i}\right)$ of the singularities $\left(D, p_{i}\right)$, where $J\left(p_{i}\right)$ denotes the Jacobian ideal of $D$ at $p_{i}$. Hence

$$
\operatorname{ker}\left(\Phi_{D}\right)=\left\{s \in H^{0}\left(D, \mathcal{O}_{D}\left(-K_{D}\right)\right): s \in J\left(p_{i}\right), \forall i\right\} .
$$

We consider the anticanonical embedding of $D$ into $\mathbb{P}^{d}$ [HW81, Corollary 4.5]. An element $s$ of $H^{0}\left(D, \mathcal{O}_{D}\left(-K_{D}\right)\right)$ can be viewed as a hyperplane section of $D \subset \mathbb{P}^{d}$, and $s \in J\left(p_{i}\right)$ means that the corresponding hyperplane section contains the scheme $\operatorname{Spec} \mathcal{O}_{D, p_{i}} / J\left(p_{i}\right)$. Therefore the claim is obviously true when $D$ is non-singular, or has one or two $A_{1}$ singularities, because in this case $s$ belongs to the Jacobian ideal if and only if the corresponding hyperplane section passes through the (two) singular point(s).

We proceed by descending induction on $d=\operatorname{deg} D$. Suppose $D^{\prime} \subset \mathbb{P}^{d}$ is a (normal, rational) del Pezzo surface of degree $d \geqslant 5$. Then there is a del Pezzo surface $D \subset \mathbb{P}^{d+1}$ of degree $d+1$ and a smooth point $p \in D$ such that the closure of the image of $D$ under the projection $\pi_{p}: \mathbb{P}^{d+1} \rightarrow-\mathbb{P}^{d}$ is $D^{\prime}$. We have

$$
H^{0}\left(D^{\prime}, \mathcal{O}_{D^{\prime}}\left(-K_{D^{\prime}}\right)\right) \simeq\left\{s \in H^{0}\left(D, \mathcal{O}_{D}\left(-K_{D}\right)\right): s(p)=0\right\} .
$$

Note that if $p$ does not belong to any line on $D$, then $S\left(D^{\prime}\right) \simeq S(D)$, and the claim follows.

Suppose now that $p$ belongs to a line $L \subset D$. If $L$ does not contain any singularities of $D$, then $\pi_{p}(L-p)$ is a new $A_{1}$ singularity on $D^{\prime}$; if $L$ contains an $A_{\lambda}$ singularity, then $\pi_{p}(L-p)$ is an $A_{\lambda+1}$ singularity; and if $L$ contains an $A_{\lambda_{1}}$ and an $A_{\lambda_{2}}$ singularity, then $\pi_{p}(L-p)$ is an $A_{\lambda_{1}+\lambda_{2}}$ singularity.

The claim now follows from the observation that

$$
\operatorname{dim}\left(\operatorname{ker} \Phi_{D^{\prime}}\right)=\operatorname{dim}\left(\operatorname{ker} \Phi_{D}\right)-1-\#(\text { lines through } p) .
$$

This completes the proof of Theorem 4.4. 


\section{Classification of CREPANT EXTREMAL CONTRACTIONS}

COROLlary 4.5. The Main Theorem holds, i.e. the contraction $\varphi$ is analytically rigid.

Proof. Suppose $D$ and $X$ are as in the Main Theorem (Theorem 4.1). Then, by Proposition 4.3 and Corollary 3.8, any two embeddings of $D$ into smooth 3 -folds with normal bundles isomorphic to $\mathcal{O}_{D}\left(K_{D}\right)$ are 2-equivalent. We also have the vanishing of $H^{1}\left(D, \mathcal{T}_{X} \otimes \mathcal{I}^{\nu} / \mathcal{I}^{\nu+1}\right)$ for any $\nu \geqslant 2$ (Theorem 4.4); thus, by Lemma 3.1 and by [HR64, Theorem 3], the Main Theorem holds.

Remark 4.6. When $D$ is non-singular, the vanishing of $H^{1}\left(D, \mathcal{T}_{X} \otimes \mathcal{I}_{D} / \mathcal{I}_{D}^{2}\right)$ is an easy consequence of the fact that $H^{1}\left(D, \mathcal{T}_{D}\right)=0$ (via the exact sequence (4.4), using Proposition 4.3 and the vanishing of $H^{1}\left(D, \mathcal{O}_{D}\right)$ ). Hence, by induction (as in the proof of Theorem 4.4), we have the vanishing of $H^{1}\left(D, \mathcal{T}_{X} \otimes \mathcal{I}_{D}^{\nu} / \mathcal{I}_{D}^{\nu+1}\right)$, for all $\nu \geqslant 2$. However, if $D$ is singular, it is not generally true that $H^{1}\left(D, \mathcal{T}_{D}\right)=0$ implies $H^{1}\left(D, \mathcal{T}_{X} \otimes \mathcal{I}_{D} / \mathcal{I}_{D}^{2}\right)=0$.

Indeed, suppose $D$ has at least one singular point. Consider the exact sequence

$$
\begin{aligned}
0 & \rightarrow \operatorname{Hom}\left(\Omega_{D}^{1}, \mathcal{I}_{D} / \mathcal{I}_{D}^{2}\right) \rightarrow \operatorname{Hom}\left(\left.\Omega_{X}^{1}\right|_{D}, \mathcal{I}_{D} / \mathcal{I}_{D}^{2}\right) \rightarrow \operatorname{Hom}\left(\mathcal{I}_{D} / \mathcal{I}_{D}^{2}, \mathcal{I}_{D} / \mathcal{I}_{D}^{2}\right) \\
\stackrel{\delta}{\rightarrow} \operatorname{Ext}^{1}\left(\Omega_{D}^{1}, \mathcal{I}_{D} / \mathcal{I}_{D}^{2}\right) & \rightarrow \operatorname{Ext}^{1}\left(\left.\Omega_{X}^{1}\right|_{D}, \mathcal{I}_{D} / \mathcal{I}_{D}^{2}\right) \rightarrow \operatorname{Ext}^{1}\left(\mathcal{I}_{D} / \mathcal{I}_{D}^{2}, \mathcal{I}_{D} / \mathcal{I}_{D}^{2}\right)
\end{aligned}
$$

obtained from (4.4). We have $\operatorname{Ext}^{1}\left(\mathcal{I}_{D} / \mathcal{I}_{D}^{2}, \mathcal{I}_{D} / \mathcal{I}_{D}^{2}\right) \simeq H^{1}\left(D, \mathcal{O}_{D}\right)=0$ and $\operatorname{Ext}^{1}\left(\left.\Omega_{X}^{1}\right|_{D}, \mathcal{I}_{D} / \mathcal{I}_{D}^{2}\right) \simeq$ $H^{1}\left(D, \mathcal{T}_{X} \otimes \mathcal{I}_{D} / \mathcal{I}_{D}^{2}\right)$ by Serre duality. Therefore we obtain

$$
\begin{aligned}
& 0 \rightarrow \operatorname{Hom}\left(\Omega_{D}^{1}, \mathcal{I}_{D} / \mathcal{I}_{D}^{2}\right) \rightarrow \operatorname{Hom}\left(\left.\Omega_{X}^{1}\right|_{D}, \mathcal{I}_{D} / \mathcal{I}_{D}^{2}\right) \rightarrow k \\
& \stackrel{\delta}{\rightarrow} \operatorname{Ext}^{1}\left(\Omega_{D}^{1}, \mathcal{I}_{D} / \mathcal{I}_{D}^{2}\right) \rightarrow H^{1}\left(D, \mathcal{I}_{X} \otimes \mathcal{I}_{D} / \mathcal{I}_{D}^{2}\right) \rightarrow 0 .
\end{aligned}
$$

Here $\delta(1)$ corresponds to the extension (4.4) that is not split, and hence it is non-zero in $\operatorname{Ext}^{1}\left(\Omega_{D}^{1}, \mathcal{I}_{D} / \mathcal{I}_{D}^{2}\right)$. Therefore $\delta$ is injective and we have

$$
0 \rightarrow k \stackrel{\delta}{\rightarrow} \operatorname{Ext}^{1}\left(\Omega_{D}^{1}, \mathcal{I}_{D} / \mathcal{I}_{D}^{2}\right) \rightarrow H^{1}\left(D, \mathcal{T}_{X} \otimes \mathcal{I}_{D} / \mathcal{I}_{D}^{2}\right) \rightarrow 0 .
$$

From the five-term exact sequence associated to the local to global spectral sequence, we have

$$
\begin{aligned}
0 & \rightarrow H^{1}\left(D, \mathcal{H o m}\left(\Omega_{D}^{1}, \mathcal{O}_{D}\right)\right) \rightarrow \operatorname{Ext}^{1}\left(\Omega_{D}^{1}, \mathcal{O}_{D}\right) \rightarrow H^{0}\left(D, \mathcal{E x t}_{\mathcal{O}_{D}}^{1}\left(\Omega_{D}^{1}, \mathcal{O}_{D}\right)\right) \\
& \rightarrow H^{2}\left(D, \mathcal{H o m}\left(\Omega_{D}^{1}, \mathcal{O}_{D}\right)\right) \rightarrow \operatorname{Ext}^{2}\left(\Omega_{D}^{1}, \mathcal{O}_{D}\right),
\end{aligned}
$$

and, as $\mathcal{H o m}\left(\Omega_{D}^{1}, \mathcal{O}_{D}\right)=\mathcal{T}_{D}$ and $H^{2}\left(D, \mathcal{T}_{D}\right)=0[\mathrm{Gr} 97 \mathrm{a}$, Lemma 5.6], we obtain

$$
0 \rightarrow H^{1}\left(D, \mathcal{T}_{D}\right) \rightarrow \operatorname{Ext}^{1}\left(\Omega_{D}^{1}, \mathcal{O}_{D}\right) \rightarrow H^{0}\left(D, \mathcal{E} x t_{\mathcal{O}_{D}}^{1}\left(\Omega_{D}^{1}, \mathcal{O}_{D}\right)\right) \rightarrow 0 .
$$

It is easy to see that $\operatorname{dim} H^{0}\left(D, \mathcal{E} x t_{\mathcal{O}_{D}}^{1}\left(\Omega_{D}^{1}, \mathcal{O}_{D}\right)\right)=\sum_{i=1}^{r} \lambda_{i}$ if $D$ has $r$ singularities, of type $A_{\lambda_{1}}$, $A_{\lambda_{2}}, \ldots, A_{\lambda_{r}}$, respectively. Therefore, if $H^{1}\left(D, \mathcal{T}_{D}\right)=0$, we obtain $\operatorname{dim} \operatorname{Ext}^{1}\left(\Omega_{D}^{1}, \mathcal{O}_{D}\right)=\sum_{i=1}^{r} \lambda_{i}$.

Now suppose that $H^{1}\left(D, \mathcal{T}_{X} \otimes \mathcal{I}_{D} / \mathcal{I}_{D}^{2}\right)=0$. Then (4.6) implies that $\operatorname{dim} \operatorname{Ext}^{1}\left(\Omega_{D}^{1}, \mathcal{O}_{D}\right)=1$, and hence $D$ can have only one singularity, of type $A_{1}$. However, this is not the case in general, and hence $H^{1}\left(D, \mathcal{T}_{X} \otimes \mathcal{I}_{D} / \mathcal{I}_{D}^{2}\right)$ does not vanish for a general singular del Pezzo surface with $H^{1}\left(D, \mathcal{I}_{D}\right)=0$.

\section{Computing the obstruction to formal equivalence: the vanishing of $H^{1}\left(D, \mathcal{T}_{D}\right)$}

Theorem 5.1. Let $D$ be a normal rational del Pezzo surface of degree $d \geqslant 5$. Then

$$
H^{1}\left(D, \mathcal{T}_{D}\right)=0 .
$$

Let $\pi: \tilde{D} \rightarrow D$ denote the minimal resolution of $D$. The proof is done below in several steps (involving $\S \S 5.1-5.3$, Lemmas 5.2-5.4, Theorem 5.5 and Corollary 5.6), by comparing the cohomology of the tangent sheaf on $D$ to the cohomology of the tangent bundle on $\tilde{D}$. 


\section{Cs. TAmás}

\subsection{The Leray spectral sequence}

As $D$ is a surface having only isolated normal singularities, $\pi_{*} \mathcal{T}_{\tilde{D}} \simeq \mathcal{T}_{D}$ [BW74, Proposition (1.2)]. We use this fact and the Leray spectral sequence

$$
E_{2}^{p, q}=H^{p}\left(D, R^{q} \pi_{*} \mathcal{T}_{\tilde{D}}\right) \Rightarrow E_{\infty}^{p+q}=H^{p+q}\left(\tilde{D}, \mathcal{T}_{\tilde{D}}\right)
$$

to compare the cohomology of $\mathcal{T}_{D}$ with that of $\mathcal{T}_{\tilde{D}}$.

The first four terms of the corresponding five-term exact sequence [God58, Theorem I.4.5.1]

$$
0 \rightarrow E_{2}^{1,0} \rightarrow E_{\infty}^{1} \rightarrow E_{2}^{0,1} \rightarrow E_{2}^{2,0} \rightarrow E_{\infty}^{2}
$$

give in our case

$$
0 \rightarrow H^{1}\left(D, \pi_{*} \mathcal{T}_{\tilde{D}}\right) \rightarrow H^{1}\left(\tilde{D}, \mathcal{T}_{\tilde{D}}\right) \rightarrow H^{0}\left(D, R^{1} \pi_{*} \mathcal{T}_{\tilde{D}}\right) \rightarrow H^{2}\left(D, \pi_{*} \mathcal{T}_{\tilde{D}}\right)
$$

With the identification $\pi_{*} \mathcal{T}_{\tilde{D}} \simeq \mathcal{T}_{D}$, and using $H^{2}\left(D, \mathcal{T}_{D}\right)=0$ [Gr97a, Lemma 5.6], we obtain

$$
0 \rightarrow H^{1}\left(D, \mathcal{T}_{D}\right) \rightarrow H^{1}\left(\tilde{D}, \mathcal{T}_{\tilde{D}}\right) \rightarrow H^{0}\left(D, R^{1} \pi_{*} \mathcal{T}_{\tilde{D}}\right) \rightarrow 0 .
$$

In order to show the vanishing of $H^{1}\left(D, \mathcal{T}_{D}\right)$, we show that $\operatorname{dim} H^{1}\left(\tilde{D}, \mathcal{T}_{\tilde{D}}\right)=\operatorname{dim} H^{0}\left(D, R^{1} \pi_{*} \mathcal{T}_{\tilde{D}}\right)$.

5.2 Local computations: $H^{0}\left(D, R^{1} \pi_{*} \mathcal{T}_{\tilde{D}}\right)$

As $D$ is a normal rational del Pezzo surface of degree $d \geqslant 5$, it has only singularities of type $A_{1}, A_{2}$, $A_{3}$ and $A_{4}$ (Lemma 2.7). Denote by $E$ the exceptional locus of its minimal resolution $\pi: \tilde{D} \rightarrow D$.

We have $H^{0}\left(D, R^{1} \pi_{*} \mathcal{T}_{\tilde{D}}\right) \simeq R^{1} \pi_{*} \mathcal{T}_{\tilde{D}}$ if regarded as complex vector spaces, as $R^{1} \pi_{*} \mathcal{T}_{\tilde{D}}$ is a skyscraper sheaf supported on the singular points of $D$. By the theorem of formal functions [Har77, Theorem III.11.1], we obtain

$$
R^{1} \pi_{*} \mathcal{T}_{\tilde{D}} \simeq \lim _{\longleftarrow} H^{1}\left(\mathcal{E}_{n},\left.\mathcal{T}_{\tilde{D}}\right|_{\mathcal{E}_{n}}\right),
$$

where $\mathcal{E}_{n}$ is the closed subscheme of $\tilde{D}$ defined by $\mathcal{I}_{E}^{n}$, where $\mathcal{I}_{E}$ is the ideal sheaf of $E$ in $\tilde{D}$.

From [BW74, (1.6)], we have the following lemma, true for any surface having only isolated rational singularities.

Lemma 5.2. If $Z$ is an effective divisor on $\tilde{D}$ supported on $E$, there is an exact sequence

$$
0 \rightarrow \mathcal{T}_{Z} \rightarrow \mathcal{T}_{\tilde{D}} \mid Z \rightarrow \bigoplus_{i=1}^{\lambda} \mathcal{N}_{E_{i} / \tilde{D}} \rightarrow 0
$$

where $E_{1}, E_{2}, \ldots, E_{\lambda}$ are the irreducible components of $E$ and $\mathcal{N}_{E_{i} / \tilde{D}}:=\mathcal{O}_{E_{i}}\left(E_{i}\right)=\mathcal{O}_{E_{i}}(-2)$ is the normal bundle of $E_{i}$ in $\tilde{D}$. (The second map of (5.2) is the sum of the compositions $\mathcal{T}_{\tilde{D}} \otimes \mathcal{O}_{Z} \rightarrow$ $\left.\mathcal{T}_{\tilde{D}} \otimes \mathcal{O}_{E_{i}} \rightarrow \mathcal{N}_{E_{i} / \tilde{D}^{\cdot}}\right)$

By the tautness of rational double point singularities [Tju68] we have that $H^{1}\left(\mathcal{T}_{Z}\right)=0$. Therefore, the long exact sequence obtained from (5.2) implies $h^{1}\left(\mathcal{E}_{n},\left.\mathcal{T}_{\tilde{D}}\right|_{\mathcal{E}_{n}}\right)=h^{1}\left(\mathcal{E}_{n}, \bigoplus_{i=1}^{\lambda} \mathcal{N}_{E_{i} / \tilde{D}}\right)$, for all $n \geqslant 0$. This shows that, if $D$ has $r$ singularities, of type $A_{\lambda_{1}}, A_{\lambda_{2}}, \ldots, A_{\lambda_{r}}$, respectively, then $h^{0}\left(D, R^{1} \pi_{*} \mathcal{T}_{\tilde{D}}\right)=\sum_{i=1}^{r} \lambda_{i}$

\subsection{Global computations: $H^{1}\left(\tilde{D}, \mathcal{T}_{\tilde{D}}\right)$}

Here we show that $\operatorname{dim} H^{1}\left(\tilde{D}, \mathcal{T}_{\tilde{D}}\right)=\sum_{i=1}^{r} \lambda_{i}$ as well, and therefore we obtain $H^{1}\left(D, \mathcal{T}_{D}\right)=0$ from the sequence (5.1).

First, we need some preliminary results relating the tangent bundle of a (smooth) surface $S$ to that of a one-point blowup of $S$.

Lemma 5.3. Let $\sigma: S^{\prime} \rightarrow S$ be a birational morphism of smooth projective surfaces and let $\mathcal{F}$ be a locally free sheaf on $S$. Then $H^{*}(S, \mathcal{F})=H^{*}\left(S^{\prime}, \sigma^{*} \mathcal{F}\right)$. 


\section{Classification of CREPANT EXTREMAl CONTRACTIONS}

Proof. The morphism $\sigma$ can be factored as the composition of blowups. Therefore it is enough to assume that $\sigma$ itself is the blowup of a point $p \in S$.

Because the sheaf $\mathcal{F}$ is locally free, the projection formula and normality of $S$ imply that $\sigma_{*} \sigma^{*} \mathcal{F} \simeq \mathcal{F}$. Therefore, by a degenerate case of the Leray spectral sequence, it is enough to show that $R^{i} \sigma_{*} \sigma^{*} \mathcal{F}=0, \forall i>0$. But again, by the projection formula and $\sigma_{*} \mathcal{O}_{S^{\prime}}=\mathcal{O}_{S}$, we can reduce this to showing that $R^{i} \sigma_{*} \mathcal{O}_{S^{\prime}}=0$, which is proven in [Har77, Proposition V.3.4].

Lemma 5.4. Let $\sigma: S^{\prime} \rightarrow S$ be the blowup of a smooth projective variety $S$ of dimension $n$ at a point $p$ and let $\mathcal{E}$ denote the exceptional locus of $\sigma$. We then have an exact sequence

$$
0 \rightarrow \mathcal{T}_{S^{\prime}} \rightarrow \sigma^{*} \mathcal{T}_{S} \rightarrow \mathcal{T}_{\mathcal{E}} \otimes \mathcal{O}_{\mathcal{E}}(\mathcal{E}) \rightarrow 0 .
$$

Proof. The first fundamental exact sequence of differentials

$$
0 \rightarrow \sigma^{*} \Omega_{S}^{1} \rightarrow \Omega_{S^{\prime}}^{1} \rightarrow \Omega_{S^{\prime} / S} \rightarrow 0
$$

gives, after taking $\mathcal{H o m}_{\mathcal{O}_{S^{\prime}}}\left(-, \mathcal{O}_{S^{\prime}}\right)$ :

$$
0 \rightarrow \mathcal{T}_{S^{\prime}} \rightarrow \sigma^{*} \mathcal{T}_{S} \rightarrow \mathcal{E} x t_{\mathcal{O}_{S^{\prime}}}^{1}\left(\Omega_{\mathcal{E}}^{1}, \mathcal{O}_{S^{\prime}}\right) \rightarrow 0,
$$

where we used $\Omega_{S^{\prime} / S} \simeq \Omega_{\mathcal{E}}^{1}$ (see for example [Kle81]).

As $\mathcal{E} x t_{\mathcal{O}_{S^{\prime}}}^{1}\left(\mathcal{O}_{\mathcal{E}}, \mathcal{O}_{S^{\prime}}\right)=\mathcal{O}_{\mathcal{E}}(\mathcal{E})$, from the conormal exact sequence

$$
0 \rightarrow \mathcal{I}_{\mathcal{E}} / \mathcal{I}_{\mathcal{E}}^{2} \rightarrow \Omega_{S^{\prime}}^{1} \mid \mathcal{E} \rightarrow \Omega_{\mathcal{E}}^{1} \rightarrow 0
$$

we obtain

$$
0 \rightarrow \mathcal{E} x t_{\mathcal{O}_{S^{\prime}}}^{1}\left(\Omega_{\mathcal{E}}^{1}, \mathcal{O}_{S^{\prime}}\right) \rightarrow \mathcal{T}_{S^{\prime}} \otimes_{\mathcal{O}_{S^{\prime}}} \mathcal{O}_{\mathcal{E}}(\mathcal{E}) \rightarrow \mathcal{O}_{\mathcal{E}}(2 \mathcal{E}) \rightarrow 0
$$

Tensoring the dual of $(5.5)$ by $\mathcal{O}_{\mathcal{E}}(\mathcal{E})$, we have

$$
0 \rightarrow \mathcal{T}_{\mathcal{E}} \otimes \mathcal{O}_{\mathcal{E}}(\mathcal{E}) \rightarrow \mathcal{T}_{S^{\prime}} \otimes_{\mathcal{O}_{S^{\prime}}} \mathcal{O}_{\mathcal{E}}(\mathcal{E}) \rightarrow \mathcal{O}_{\mathcal{E}}(2 \mathcal{E}) \rightarrow 0
$$

Comparing the last two exact sequences, we obtain the desired result.

In particular, if the variety $S$ in Lemma 5.4 is a smooth surface, the exact sequence (5.3) translates to

$$
0 \rightarrow \mathcal{T}_{S^{\prime}} \rightarrow \sigma^{*} \mathcal{T}_{S} \rightarrow \mathcal{O}_{\mathcal{E}}(1) \rightarrow 0 .
$$

We can use the associated long exact sequence and Lemma 5.3 to obtain

$$
\begin{aligned}
H^{0}\left(S^{\prime}, \mathcal{T}_{S^{\prime}}\right) & =\operatorname{ker}\left(H^{0}\left(S, \mathcal{T}_{S}\right) \rightarrow H^{0}\left(\mathcal{E}, \mathcal{O}_{\mathcal{E}}(1)\right)\right) \\
\operatorname{dim} H^{1}\left(S^{\prime}, \mathcal{T}_{S^{\prime}}\right) & =h^{0}\left(S^{\prime}, \mathcal{T}_{S^{\prime}}\right)-h^{0}\left(S, \mathcal{T}_{S}\right)+2+h^{1}\left(S, \mathcal{T}_{S}\right) .
\end{aligned}
$$

Therefore Lemma 5.4 gives us a tool to compute $H^{1}\left(\tilde{D}, \mathcal{T}_{\tilde{D}}\right)$ step-by-step, blowing up one point at a time.

TheOREM 5.5. Let $\Sigma$ be a set of (possibly infinitely near) points of $\mathbb{P}^{2}$ in almost general position. Suppose $|\Sigma| \leqslant 3$. Let $\sigma: S \rightarrow \mathbb{P}^{2}$ be the blowup of center $\Sigma$. Let $p \in S$ be a point such that $\Sigma^{\prime}=\Sigma \cup\{p\}$ is in almost general position and let $\sigma^{\prime}: S^{\prime} \rightarrow S$ be the blowup of $S$ at $p$. Denote by $\tilde{\mathcal{E}}$ the union of all curves with negative self-intersection on $S$. Then we have the following:

i) If $p \notin \tilde{\mathcal{E}}$, then $h^{0}\left(S^{\prime}, \mathcal{T}_{S^{\prime}}\right)=h^{0}\left(S, \mathcal{T}_{S}\right)-2$.

ii) If $p$ is contained in a single $(-1)$-curve, then $h^{0}\left(S^{\prime}, \mathcal{T}_{S^{\prime}}\right)=h^{0}\left(S, \mathcal{T}_{S}\right)-1$.

iii) If $p$ is the intersection point of two $(-1)$-curves, then $h^{0}\left(S^{\prime}, \mathcal{T}_{S^{\prime}}\right)=h^{0}\left(S, \mathcal{T}_{S}\right)$.

The proof of Theorem 5.5 follows after Corollary 5.6 and Remark 5.7. 


\section{Cs. TAMÁs}

Corollary 5.6. If $D$ is a normal rational del Pezzo surface of degree $d \geqslant 5$, with $r$ singularities, of type $A_{\lambda_{1}}, A_{\lambda_{2}}, \ldots, A_{\lambda_{r}}$, respectively, and $\pi: \tilde{D} \rightarrow D$ is its minimal resolution, then we have $\operatorname{dim} H^{1}\left(\tilde{D}, \mathcal{T}_{\tilde{D}}\right)=\sum_{i=1}^{r} \lambda_{i}$, and hence $H^{1}\left(D, \mathcal{T}_{D}\right)=0$.

Proof. Note that $h^{1}\left(S^{\prime}, \mathcal{T}_{S^{\prime}}\right)-h^{1}\left(S, \mathcal{T}_{S}\right)$ counts the number of $(-2)$-curves that appear on $S^{\prime}$ after the blowup $\sigma^{\prime}$, thus the corollary follows.

This concludes the proof of Theorem 5.1.

Remark 5.7. As $H^{1}\left(D, \mathcal{T}_{D}\right)$ is the tangent space of the locally trivial deformations of $D$, we can conclude that all locally trivial deformations of a normal rational del Pezzo surface of degree $d \geqslant 5$ are trivial. This was to be expected, as configurations of at most four points in almost general position on $\mathbb{P}^{2}$ (giving the same del Pezzo surface) have no moduli.

Proof of Theorem 5.5. Note that, if the surface $S$ is obtained by successive blowups of (possibly infinitely near) points on $\mathbb{P}^{2}$, then we can regard $H^{0}\left(S, \mathcal{T}_{S}\right)$ as a subspace of $H^{0}\left(\mathbb{P}^{2}, \mathcal{T}_{\mathbb{P}^{2}}\right)$. We prove the theorem by blowing up one point at a time and explicitly computing the cohomologies involved.

Case 0: Explicit computation of $H^{0}\left(\mathbb{P}^{2}, \mathcal{T}_{\mathbb{P}^{2}}\right)$. It is well known that $\operatorname{dim} H^{0}\left(\mathbb{P}^{2}, \mathcal{T}_{\mathbb{P}^{2}}\right)=8$ and $\operatorname{dim} H^{1}\left(\mathbb{P}^{2}, \mathcal{T}_{\mathbb{P}^{2}}\right)=0$. Here we compute a basis for $H^{0}\left(\mathbb{P}^{2}, \mathcal{T}_{\mathbb{P}^{2}}\right)$ in local coordinates.

Fix the homogeneous coordinates $\left[x_{0}: x_{1}: x_{2}\right]$ on $\mathbb{P}^{2}$. Then on the affine open $U_{0}=\left\{x_{0} \neq 0\right\}$ we have local coordinates $x:=x_{1} / x_{0}$ and $y:=x_{2} / x_{0}$. Around $p=[1: 0: 0], \mathcal{T}_{\mathbb{P}^{2}}$ is generated by the vectors $\partial_{x}=\partial / \partial x$ and $\partial_{y}=\partial / \partial y$; more precisely, $\left.\mathcal{T}_{\mathbb{P}^{2}}\right|_{U_{0}}=\mathbb{C}[x, y] \partial_{x}+\mathbb{C}[x, y] \partial_{y}$.

Claim. With the above notations, $H^{0}\left(\mathbb{P}^{2}, \mathcal{T}_{\mathbb{P}^{2}}\right)$ has a basis given by $v_{1}=\partial_{x}, v_{2}=\partial_{y}, v_{3}=x \partial_{x}$, $v_{4}=x \partial_{y}, v_{5}=y \partial_{x}, v_{6}=y \partial_{y}, v_{7}=x^{2} \partial_{x}+x y \partial_{y}$, and $v_{8}=x y \partial_{x}+y^{2} \partial_{y}$ on $U_{0}$.

Proof. We have the dual of the Euler sequence,

$$
0 \rightarrow \mathcal{O}_{\mathbb{P}^{2}} \rightarrow \mathcal{O}_{\mathbb{P}^{2}}(1)^{3} \rightarrow \mathcal{T}_{\mathbb{P}^{2}} \rightarrow 0,
$$

where $\mathcal{O}_{\mathbb{P}^{2}}(1)^{3} \rightarrow \mathcal{T}_{\mathbb{P}^{2}}$ is (locally) given by

$$
\left(s_{0}, s_{1}, s_{2}\right) \mapsto \frac{s_{1} x_{0}-s_{0} x_{1}}{x_{0}^{2}} \partial_{x}+\frac{s_{2} x_{0}-s_{0} x_{2}}{x_{0}^{2}} \partial_{y} .
$$

Writing out the generators of $H^{0}\left(\mathbb{P}^{2}, \mathcal{O}_{\mathbb{P}^{2}}(1)^{3}\right)$, the claim follows.

Using this explicit description of $H^{0}\left(\mathbb{P}^{2}, \mathcal{T}_{\mathbb{P}^{2}}\right)$, the computations are straightforward. We illustrate it in two cases: blowing up one point, and blowing up two infinitely near points on $\mathbb{P}^{2}$.

Case 1: Blowing up a point. Let $\sigma_{1}: S_{1} \rightarrow \mathbb{P}^{2}$ be the blowup of the point $(x, y)=(0,0) \in U_{0}$, and let $\mathcal{E}$ denote the exceptional locus. On the affine open $V_{0}=\operatorname{Spec} \mathbb{C}[x, s]$, where $x=x, y=x s$, $\mathcal{E}$ is defined by the equation $\{x=0\}$ and the sequence (5.3) is

$$
0 \rightarrow \mathbb{C}[x, s] \partial_{x} \oplus \mathbb{C}[x, s] \partial_{s} \rightarrow \mathbb{C}[x, s] \partial_{x} \oplus \mathbb{C}[x, s] \partial_{y} \rightarrow \frac{1}{x} \mathbb{C}[s] \partial_{s} \rightarrow 0,
$$

where the first map is given by

$$
\partial_{x} \mapsto \partial_{x}+s \partial_{y}, \quad \partial_{s} \mapsto x \partial_{y}
$$

while the second map is given by

$$
f(x, s) \partial_{x}+g(x, s) \partial_{y} \mapsto \frac{g(0, s)-s f(0, s)}{x} \partial_{s} .
$$

Note that the images of $v_{1}=\partial_{x}$ and $v_{2}=\partial_{y}\left(-(s / x) \partial_{s}\right.$ and $(1 / x) \partial_{s}$ respectively) generate the global sections of $\mathcal{O}_{\mathcal{E}}(1)$. 
As $y=x s$, it is easy to check that the images of $v_{3}, v_{4}, v_{5}, v_{6}, v_{7}$ and $v_{8}$ in $H^{0}\left(\mathcal{E}, \mathcal{O}_{\mathcal{E}}(1)\right)$ are all zero, and hence

$$
H^{0}\left(S_{1}, \mathcal{T}_{S_{1}}\right)=\operatorname{ker}\left(H^{0}\left(\mathbb{P}^{2}, \mathcal{T}_{\mathbb{P}^{2}}\right) \rightarrow H^{0}\left(\mathcal{E}, \mathcal{O}_{\mathcal{E}}(1)\right)\right) \simeq\left\langle v_{2}, v_{3}, v_{5}, v_{6}, v_{7}, v_{8}\right\rangle
$$

This shows that $\operatorname{dim} H^{0}\left(S_{1}, \mathcal{T}_{S_{1}}\right)=6$. In terms of $\partial_{x}$ and $\partial_{s}$, we have

$$
\begin{aligned}
& v_{3}=x \partial_{x}-s \partial_{s}, \\
& v_{4}=\partial_{s}, \\
& v_{5}=x s \partial_{x}-s^{2} \partial_{s}, \\
& v_{6}=s \partial_{s}, \\
& v_{7}=x^{2}(1+s) \partial_{x}-x s(1+s) \partial_{s}, \\
& v_{8}=x s(1+s) \partial_{s} .
\end{aligned}
$$

Case 2: Blowing up two infinitely near points of $\mathbb{P}^{2}$. Let $S_{2}$ be the blowup of the point $(x, s)=$ $(0,0) \in S_{1}$ (corresponding to the direction given by the line $\{y=0\}$ in $\mathbb{P}^{2}$ ). Let $\mathcal{E}^{\prime}$ denote the exceptional locus. As in the previous case, we see that the image of $v_{4}=\partial_{s}$ is a global section of $H^{0}\left(\mathcal{E}^{\prime}, \mathcal{O}_{\mathcal{E}^{\prime}}(1)\right)$, while the images of $v_{3}, v_{5}, v_{6}, v_{7}$ and $v_{8}$ are all zero. Hence $\operatorname{dim} H^{0}\left(S_{2}, \mathcal{T}_{S_{2}}\right)=5$.

For the rest of the cases, similar computations can be carried out, and we leave them to the reader. Thus the proof of Theorem 5.5 is finished.

\section{Example of a $K$-trivial contraction}

Let $\varphi: X \rightarrow Y$ be a $K$-trivial birational extremal contraction of a smooth projective 3 -fold $X$, contracting a divisor $D \subset X$ to a point $q \in Y$. Suppose $D$ is a normal rational del Pezzo surface of degree $d \geqslant 5$. By our Main Theorem, in order to know the analytic structure of the contraction $\varphi$, it is sufficient to have one example for each possible exceptional divisor with the prescribed normal bundle.

In the following, we construct an example of embedding a normal rational (singular) del Pezzo surface $D$ of degree 7 into a smooth 3 -fold $X$ with the prescribed normal bundle $\mathcal{O}_{D}\left(K_{D}\right)$, and hence, by Fujiki's contraction theorem (see Theorem 6.1 below), obtain an analytic contraction of $D$ (i.e. a holomorphic map $\varphi: X \rightarrow Y$ onto a normal complex space $Y$ that contracts $D$ to a point $q \in Y$ ). Similar constructions can be carried out for each possible exceptional divisor $D$ (any normal rational del Pezzo surface of degree $d \geqslant 5$ ).

It should be noted that, if $D$ is non-singular, the embedding of $D$ as the zero-section into the total space of $\mathcal{O}_{D}\left(K_{D}\right)$ gives such an example. It is tempting to consider a similar approach in the singular case, by considering the zero-section embedding of the minimal resolution $\tilde{D}$ of $D$ into the total space $\tilde{X}$ of $\mathcal{O}_{\tilde{D}}\left(K_{\tilde{D}}\right)$ and then flopping the $(-2)$-curves of $\tilde{D}$. However, while any $(-2)$-curve on $\tilde{D}$ is a $(0,-2)$-curve on $\tilde{X}$, these curves are not isolated, and hence we cannot flop them [Rei83]. Therefore we take a different approach.

Let $D$ be a normal rational del Pezzo surface of degree $d=7$. First we construct a family $X \rightarrow \mathbb{A}^{1}$ such that $X$ is non-singular, the central fiber $X_{0}$ is isomorphic to $D$, and the general fibers of the family are non-singular del Pezzo surfaces of degree $d=7=\operatorname{deg} D$. We will construct the family $X \subset \mathbb{P}^{7} \times \mathbb{A}^{1}$ as the closure of a family of blowups of two distinct points on $\mathbb{P}^{2}$. We may assume that the minimal resolution $\tilde{D}$ of $D$ is isomorphic to the blowup of the infinitely near points $p_{1}=[1: 0: 0] \in \mathbb{P}^{2}$ and $p_{2}=[1: 0] \in \mathbb{P}\left(T_{p_{1}} \mathbb{P}^{2}\right)$.

Consider the curve $\mathcal{C}=\left\{x_{1}^{2}-t\left(x_{1}+x_{0}\right)=0, x_{2}=0\right\} \subset \mathbb{P}^{2} \times \mathbb{A}^{1}$. Over any $t \neq 0, \mathcal{C}$ has two points, while over $t=0$ it has a double point. The curve $\mathcal{C}$ defines a map $\Phi=\left\{\varphi_{t}\right\}_{t}: \mathbb{P}^{2} \times \mathbb{A}^{1}-\rightarrow \mathbb{P}^{7} \times \mathbb{A}^{1}$ 


\section{Cs. TAMÁs}

with base-locus $\mathcal{C}$, where $\varphi_{t}: \mathbb{P}^{2} \rightarrow \mathbb{P}^{7}$ is given by

$\left[x_{0}: x_{1}: x_{2}\right]$

$$
\longmapsto\left[x_{1}^{3}-t x_{0}^{2} x_{1}-t^{2} x_{0}^{3}-t^{2} x_{0}^{2} x_{1}: x_{2}^{3}: x_{0}^{2} x_{2}: x_{0} x_{1}^{2}-t x_{0}^{3}-t x_{0}^{2} x_{1}: x_{0} x_{2}^{2}: x_{1}^{2} x_{2}: x_{1} x_{2}^{2}: x_{0} x_{1} x_{2}\right] .
$$

Let $\mathcal{X}$ be the blowup of $\mathbb{P}^{2} \times \mathbb{A}^{1}$ with center $\mathcal{C}$. Then $\varphi_{\left|-K_{X / \mathbb{A}^{1}}\right|}$ will define an embedding of $\mathcal{X}$ into $\mathbb{P}^{7} \times \mathbb{A}^{1}$. The ideal $\mathcal{I}$ defining $X$ in this embedding is [GS02]

$$
\begin{aligned}
\mathcal{I}= & \left(g e-b h,-h f+g d+t h^{2}+t c h,-h e+g c, f^{2}-g a-t^{2} h^{2}-t h^{2}-t^{2} c h, e f-h g,\right. \\
& f d-a h+t h d, f c-h^{2},-g^{2}+b f, d e-h^{2}+t c^{2}+t c h, e a-h f+t^{2} c h+t^{2} c^{2}+t c h, \\
& \left.b d-h g+t e h+t c e,-e^{2}+c b, c a-d h-t c d, b a-f g+t^{2} e h+t^{2} c e+t c g\right) .
\end{aligned}
$$

For each $t, \mathcal{I}$ defines a surface $D_{t}$ in $\mathbb{P}^{7}$. It can be verified by direct computation that the total space $X$ of the family $\left\{D_{t}\right\}_{t \in \mathbb{A}^{1}}$ is non-singular and that $D_{t}$ is non-singular (actually a smooth del Pezzo surface of degree 7) except in the cases of $D_{0}=D$ and $D_{-4}$, which are singular del Pezzo surfaces. In fact, for $t \neq 0,-4$, the surface $D_{t}$ is the image (via the anticanonical embedding) in $\mathbb{P}^{7}$ of the blowup of the points $\left[1: \lambda_{1}: 0\right]$ and $\left[1: \lambda_{2}: 0\right]$ on $\mathbb{P}^{2}$, where $\lambda_{1}$ and $\lambda_{2}$ are the roots of the equation $u^{2}=t(u+1)$.

Since $D$ is a fiber of the family $X$, its normal bundle $\mathcal{N}_{D / X} \simeq \mathcal{O}_{D}(D)$ in $\mathcal{X}$ is numerically trivial.

In order to make the normal bundle isomorphic to $\mathcal{O}_{D}\left(K_{D}\right)$, as required for a $K$-trivial contraction, we proceed as follows.

Let $C \in\left|-K_{D}\right|$ be a general member avoiding the singular point of $D$ (it exists, because $\left|-K_{D}\right|$ is very ample). Let $X$ be the blowup of $C$ on $X, E$ the exceptional locus, $D^{\prime}$ the strict transform of $D$, and $C^{\prime}:=\left.E\right|_{D^{\prime}} \simeq C$. Because $\left.K X\right|_{D}$ and $K_{D}$ are numerically equivalent, it follows that $K_{X}$ is numerically trivial on $D^{\prime}$. Therefore $\mathcal{N}_{D^{\prime} / X}$ is numerically equivalent to $\mathcal{O}_{D^{\prime}}\left(K_{D^{\prime}}\right)$, and so, as in $\S 2, \mathcal{N}_{D^{\prime} / X} \simeq \mathcal{O}_{D^{\prime}}\left(K_{D^{\prime}}\right)$. Therefore we succeeded in embedding the del Pezzo surface $D \simeq D^{\prime}$ into a non-singular 3-fold $X$ such that its normal bundle is isomorphic to the canonical sheaf of $D$. We can now apply the following theorem [Fuj74, Theorem 2].

Theorem 6.1 (Fujiki's Contraction Theorem). Let $X$ be a complex space, $A \subset X$ an effective Cartier divisor, $B$ another complex space, and $f: A \rightarrow B$ a surjective holomorphic map. Assume that

1) the conormal bundle $\mathcal{N}_{A / X}^{*}$ is $f$-positive, and that

2) $R^{1} f_{*}\left(\mathcal{N}_{A / X}^{* \nu}\right)=0$, for all $\nu>0$.

Then there exists a modification $\psi: X \rightarrow Y$ with $\left.\psi\right|_{A}=f$. Moreover, $\psi_{*}(\mathcal{L}) \simeq \mathcal{O}_{Y}$, where the coherent sheaf $\mathcal{L}$ is defined by

$$
0 \rightarrow \mathcal{L} \rightarrow \mathcal{O}_{X} \rightarrow \mathcal{O}_{A} / \operatorname{im}\left(f^{*} \mathcal{O}_{B} \rightarrow \mathcal{O}_{A}\right) \rightarrow 0 .
$$

In our case $A:=D^{\prime} \simeq D, B$ is a point (hence $\left.\mathcal{L} \simeq \mathcal{O}_{X}\right)$ and we have $R^{1} f_{*}\left(\mathcal{N}_{A / X}^{* \nu}\right) \simeq$ $H^{1}\left(D, \mathcal{O}_{D}\left(\nu K_{D}\right)\right)=0$ for all $\nu$ (see Proposition 2.8).

Therefore we have an analytic modification $\psi: X \rightarrow Y$ contracting $D$ to a point $q \in Y$, such that $\left.\psi\right|_{X-D}: X-D \simeq Y-q$, and $\psi_{*} \mathcal{O}_{X}=\mathcal{O}_{Y}$.

Remark 6.2. A similar result is true in the category of algebraic spaces, based on a contraction theorem due to Artin [Art69b, Corollary (6.10)].

However, Fujiki's theorem does not guarantee a contraction in the algebraic category (i.e. the existence of a morphism $\varphi: X \rightarrow Y$ of algebraic varieties contracting $D$ to a point). At the present we do not know how to construct in general algebraic morphisms that contract a singular del Pezzo 


\section{Classification of CREPANT EXTREMAl CONTRACTIONS}

surface embedded into a smooth 3 -fold with the prescribed normal bundle to a point, i.e. how to obtain a $K$-trivial morphism $\varphi: X \rightarrow Y$ onto a normal projective variety $Y$ that contracts $D$ to a point $q \in Y$. For a non-singular $D$, the contraction of the zero-section of the total space of the normal bundle $\mathcal{N}_{D / X}$ provides such an example. Although this gives a $K$-trivial contraction, it is not extremal. Namikawa constructs an example of a $K$-trivial extremal contraction [Nam97, Example 1] with exceptional divisor $D$ a non-singular del Pezzo surface of degree 6 (i.e. a smooth cubic).

\section{ACKNowledGements}

The author is grateful to K. Matsuki for his advice and encouragement. I would also like to thank M. Gross and J. Kollár for helpful comments, and M. Reid for his suggestions on improving the content and presentation of the paper.

\section{REFERENCES}

Art69a M. Artin, The implicit function theorem in algebraic geometry, in Algebraic geometry (Internat. Colloq., Tata Inst. Fundam. Res.) (Oxford University Press, Oxford, 1969), 13-34.

Art69b M. Artin, Algebraization of formal moduli. I, in Global analysis (Papers in Honor of K. Kodaira) (University of Tokyo Press, Tokyo, 1969), 21-71.

BW74 D. M. Burns Jr. and J. M. Wahl, Local contributions to global deformations of surfaces, Invent. Math. 26 (1974), 67-88.

Fuj74 A. Fujiki, On the blowing down of analytic spaces, Publ. Res. Inst. Math. Sci. 10 (1974/75), 473-507.

God58 R. Godement, Topologie algébrique et théorie des faisceaux (Hermann, Paris, 1958).

Gr97a M. Gross, Deforming Calabi-Yau threefolds, Math. Ann. 308 (1997), 187-220.

Gr97b M. Gross, Primitive Calabi-Yau threefolds, J. Differential Geom. 45 (1997), 288-318.

Gra62 H. Grauert, Über Modifikationen und exzeptionelle analytische Mengen, Math. Ann. 146 (1962), $331-368$.

Gro61 A. Grothendieck, Éléments de géométrie algébrique. III. Étude cohomologique des faisceaux cohérents I, Publ. Math. Inst. Hautes Études Sci., 11 (1961).

Gro64 A. Grothendieck, Éléments de géométrie algébrique. IV. Étude locale des schémas et des morphismes de schémas I, Publ. Math. Inst. Hautes Études Sci., 20 (1964).

Gro67a A. Grothendieck, Éléments de géométrie algébrique. IV. Étude locale des schémas et des morphismes de schémas IV, Publ. Math. Inst. Hautes Études Sci., 32 (1967).

Gro67b A. Grothendieck, Fondements de la géométrie algébrique (Secrétariat Mathématique, 1967).

GS02 D. R. Grayson and M. E. Stillman, Macaulay 2 (a software system for research in algebraic geometry), available at http://www.math.uiuc.edu/Macaulay2 (2002).

Har77 R. Hartshorne, Algebraic Geometry (Springer, Berlin, 1977).

HR64 H. Hironaka and H. Rossi, On the equivalence of imbeddings of exceptional complex spaces, Math. Ann. 156 (1964), 313-333.

HW81 H. Hidaka and H. Watanabe, Normal Gorenstein surfaces with ample anti-canonical divisor, Tokyo J. Math. 4 (1981), 319-330.

Kle81 S. L. Kleiman, Multiple-point formulas. I. Iteration, Acta Math. 147 (1981), 13-49.

Kol91 J. Kollár, Flips, flops, minimal models, etc., in Surveys in differential geometry (International Press, Boston, MA, 1991), 113-199.

Lau73 H. Laufer, Taut two-dimensional singularities, Math. Ann. 205 (1973), 131-164.

Mat01 K. Matsuki, An introduction to Mori's program (Springer, Berlin, 2001).

Mor82 S. Mori, Threefolds whose canonical bundles are not numerically effective, Ann. of Math. (2) 116 (1982), 133-176. 


\section{Classification of CREPANT EXTREMAl CONTRACTIONS}

MY82 J. N. Mather and S. T. Yau, Classification of isolated hypersurface singularities by their moduli algebras, Invent. Math. 69 (1982), 243-251.

Nam97 Y. Namikawa, Deformation theory of Calabi-Yau threefolds and certain invariants of singularities, J. Algebraic Geom. 6 (1997), 753-776.

Rei75 M. Reid, Elliptic Gorenstein surface singularities (unpublished, 1975).

Rei79 M. Reid, Canonical 3-folds, in Journées de Géometrie Algébrique d'Angers, Juillet 1979 (Sijthoff \& Noordhoff, Leyden, 1980), 273-310.

Rei83 M. Reid, Minimal models of canonical 3-folds, in Algebraic varieties and analytic varieties (North-Holland, Amsterdam, 1983), 131-180.

Rei94 M. Reid, Nonnormal del Pezzo surfaces, Publ. Res. Inst. Math. Sci., 30 (1994), 695-727.

Tju68 G. Tjurina, On the tautness of rationally contractible curves on a surface, Math. USSR Izv. 2 (1968), 907-934.

Wil97 P. M. H. Wilson, Symplectic deformations of Calabi-Yau threefolds, J. Differential Geom. 45 (1997), 611-637.

Csilla Tamás ctamas@math.uga.edu

Department of Mathematics, University of Georgia, Athens, GA 30602-7403, USA 